\title{
Anthocyanin Regulatory/Structural Gene Expression in Phalaenopsis
}

\author{
Hongmei Ma and Margaret Pooler \\ U.S. Department of Agriculture/Agricultural Research Service, U.S. National Arboretum, Floral and \\ Nursery Plants Research Unit, 3501 New York Avenue, NE, Washington, DC 20002 \\ Robert Griesbach ${ }^{1}$ \\ U.S. Department of Agriculture/Agricultural Research Service, U.S. National Arboretum, Floral and \\ Nursery Plants Research Unit, 10300 Baltimore Avenue, Building 003, Beltsville, MD 20705
}

\begin{abstract}
AdDitional INDEX words. $M y b, M y c, W d, C h s, D f r$, orchid
Abstract. Anthocyanin biosynthesis requires the coordinated expression of $\mathrm{Myc}, \mathrm{Wd}, \mathrm{Chs}, \mathrm{Dfr}$, and $\mathrm{Myb}$. Chs and $\mathrm{Dfr}$ are structural genes, while $M y c, M y b$, and $W d$ are regulatory genes. Reverse transcription polymerase chain reaction was used to measure the expression of these genes in Phalaenopsis amabilis and Phalaenopsis schilleriana. P. amabilis expresses an albescent phenotype with petals and sepals that are anthocyanin free, while $P$. schilleriana has a wild-type phenotype with anthocyanin-containing petals and sepals. As expected, the petals and sepals of $P$. schilleriana expressed high levels of $C h s$ and $D f r$. The petals and sepals of $P$. amabilis expressed high levels of $C h s$ and very low levels of $D f r$. In $P$. amabilis and $P$. schilleriana, anthocyanin-specific $M y c$ and $W d$ were expressed; however, $M y b$ specific for anthocyanin biosynthesis were undetectable in $P$. amabilis. This suggests that the absence of $M y b$ expression was responsible for the lack of dihydroflavonol 4-reductase and results in the absence of anthocyanin pigmentation in $P$. amabilis petals and sepals. This was confirmed by particle bombardment of $P$. amabilis petals with functional $M y b s$ isolated from $P$. schilleriana. Comparisons of anthocyanin-related $M y b$ gene expression between $P$. schilleriana and $\boldsymbol{P}$. amabilis are between genetically different species. Phalaenopsis 'Everspring Fairy' expresses a harlequin phenotype with white petals and sepals containing large anthocyanin sectors. Harlequin flowers are ideal to evaluate anthocyanin-related $M y b$ gene expression within genetically identical but differently pigmented tissue. High levels of anthocyanin-specific $M y b$ and $D f r$ transcripts were present in the purple, but not in the white, sectors of Phalaenopsis 'Everspring Fairy' petals and sepals. There was no differential expression of $C h s, W d$, and $M y c$ between the purple and white sectors. These results are in agreement with the results from $P$. amabilis and $P$. schilleriana.
\end{abstract}

Anthocyanin biosynthesis was first characterized in Zea mays and was demonstrated to be predominantly regulated at the transcription level by two families of regulatory factors, R-like MYC (R, B, Lc, Sn) and R2R3-MYB (C1/Pl) proteins (Brevitz et al., 2000; Chandler et al., 1989; Cone et al., 1986; Grotewold et al., 2000; Hernandez et al., 2004; Irani et al., 2003; Ludwig et al., 1989; Paz-Ares et al., 1987; Radicella et al., 1991; Sainz et al., 1997; Zhang et al., 2000). The defining characteristic of the MYC family is the presence of a conserved basic helixloop-helix (bHLH) bipartite domain consisting of a presumed DNA-binding basic region and a protein-protein dimerization motif. MYB proteins are defined by the presence of a conserved DNA-binding domain that is generally formed by one to three imperfect amino acid motif(s) (R1, R2, and R3). Each repeat encodes three $\alpha$-helices, with the second and third helices forming a helix-turn-helix (HTH) structure when bound to DNA. The $Z$. mays anthocyanin regulatory genes $L c$ and $C l$ were the first plant MYC and MYB genes, respectively, to be isolated.

Recently, WD40 repeat (WDR) proteins were also recognized as part of the anthocyanin regulatory network (deVetten et al., 1997; Smith, 1999). They comprise a 40-residue WD

Received for publication 19 Sept. 2008. Accepted for publication 3 Nov. 2008. This work was supported by a Cooperative Research and Development Agreement (58-3K95-5-1074) between Kerry's Bromeliad Nursery, Inc., McCorkle Nurseries, Inc., and Agricultural Research Service.

Mention of trade names or commercial products in this publication is solely for the purpose of providing specific information and does not imply recommendation or endorsement by the U.S. Department of Agriculture.

${ }^{1}$ Corresponding author. E-mail: robert.griesbach@ars.usda.gov. motif core region that contains the glycine-histidine $(\mathrm{GH})$ dipeptide at the $\mathrm{N}$-terminus and the tryptophan-aspartate (WD) dipeptide at the C-terminus. The WD motif forms part of the WDR protein, a conserved $\beta$-propeller structure that acts as a stable surface to facilitate protein-protein interactions. Several plant WDR proteins involved in the anthocyanin pathway have been isolated (e.g., ZmPAC1, PhAN11, and AtTTG1).

Although R-like MYC proteins contain a DNA-binding region, thus far, there is no evidence that they directly bind DNA. R-like MYC proteins were shown to physically interact with C1 MYB proteins to coactivate anthocyanin synthesis by cooperatively binding to the promoters of the biosynthetic genes. Interactions between R-like MYC and WD40 proteins were also found. Based on this evidence (Baudry et al., 2006; Hartmann et al., 2005; Hernandez et al., 2004; Ramsay and Glover, 2005; Zimmermann et al., 2004), a working hypothesis was proposed for activation of anthocyanin structural genes by the triad of MYB, MYC, and WDR proteins (Ramsay and Glover, 2005). In a functional MYB-MYC-WD complex, the MYB factor binds directly to the cis-element of the structural gene, while R-like MYC might bind indirectly via a hypothetical R interaction protein (RIP). R-like MYC is centered in the complex that interacts with a MYB factor with WD proteins on its sides. Together, they activate the entire set of anthocyanin biosynthesis genes.

Six enzymes are generally involved in the anthocyanin biosynthesis pathway: chalcone synthase (CHS), chalcone isomerase $(\mathrm{CHI})$, flavanone 3-hydroxylase $(\mathrm{F} 3 \mathrm{H})$, dihydroflavonol 
4-reductase (DFR), anthocyanidin synthase (ANS), and UDPglucose-flavonoid 3-O-glucosyltransferase (UFGT). CHS is the first and key regulatory enzyme of flavonoid biosynthesis, and DFR is the first committed enzyme of anthocyanin biosynthesis in the flavonoid pathway (Griesbach, 2005).

We previously confirmed that the albescent Phalaenopsis amabilis phenotype (white petals and sepals with an anthocyanin-pigmented labellum) could be complemented by transient expression of $Z$. mays $L c$ and $C 1$ regulatory genes (Griesbach and Klein, 1993; Ma et al., 2008). To further our understanding of the regulation of the anthocyanin biosynthesis pathway in Phalaenopsis, we compared the expression of the triad of anthocyanin regulatory proteins (WD40, MYC, and MYB) and two structural genes ( $C h s$ and $D f r$ ) between species with anthocyanin-free petals ( $P$. amabilis) and anthocyanin-containing petals (Phalaenopsis schilleriana).

\section{Materials and Methods}

Plant material. Several commercial white-flowered P. amabilis hybrids (Kerry's Bromeliad Nursery, Homestead, FL), purple-spotted Phalaenopsis 'Everspring Fairy', and purple-flowered $P$. schilleriana were used in this study. $P$. amabilis expresses an albescent phenotype with anthocyanin-free petals and sepals and an anthocyanin-pigmented labellum (Griesbach and Klein, 1993). Phalaenopsis 'Everspring Fairy' expresses a harlequin phenotype with white sepals, one or two large anthocyanin spots on white petals, and an anthocyanin-pigmented labellum (Tang and Chen, 2007). P. schilleriana expresses the wild-type phenotype with solid purple petals, sepals, and labellum. All plants were grown in commercial orchid greenhouses until flowering. Flowering plants were then held in the laboratory for the duration of the study. The flower buds of $P$. amabilis and P. schilleriana harvested for RNA isolation were 1.0 to $1.5 \mathrm{~cm}$ in length. Flower petals of Phalaenopsis 'Everspring Fairy' were harvested when flowers were just fully open.

RNA EXTRACTION, PRIMER DESIGN, REVERSE TRANSCRIPTION (RT) POLYMERASE CHAIN REACTION (PCR), AND SUBCLONING. Total RNAs of $P$. amabilis and $P$. schilleriana were extracted from $100 \mathrm{mg}$ of flower bud petals using the RNeasy Plant Mini Kit (Qiagen, Valencia, CA). White and purple sections from petals of Phalaenopsis 'Everspring Fairy' were dissected and total RNAs were extracted from $100 \mathrm{mg}$ of dissected petals using the same kit. Residual genomic DNA contamination was removed from RNA preparations using the DNA-free ${ }^{\mathrm{TM}}$ kit (Ambion, Austin, TX). Primers for $M y b, M y c, W d, C h s$, and $D f r$ genes were designed based on the conserved region of published sequences:

$M y b$ : forward 5'-GGGAAGAGCTGCCGGCT-3'; reverse 5'TCCAGTAGTTCTTGATCTC-3' [Dendrobium sp. (AF485892, AF485893, AF485895, AF485899, AF485900, and AF485901), Gerbera hybrid (AJ554697), Triticum aestivum (AB252146), and $Z$. mays (M37153)]

Myc: forward 5'-TGGAGCTATGCCATTTTCTGGT-3'; reverse 5' CTGAATAGATGCACTCTTGGC-3' [Z. mays (M26227, X60706, and X57276), Petunia ×hybrida (AF020545 and AF260918), Cornus canadensis (AY493694), Perilla frutescens (AB024050), and Antirrhinum majus (M84913)]

Wd: forward 5' -GTGTTTGCTTCGGTCTCTGCTGA-3'; reverse 5' GCAGACCACTGAAGCTGATTAAT-3' [Matthiola incana (AJ586862), Arabidopsis thaliana (NM_101162), Gossypium hir- sutum (AF530911), Malus $\times$ domestica (AF220203), P. frutescens (AB059642), Ipomoea purpurea (AB232777), P. ×hybrida (U94748), and Z. mays (AY115485)]

Chs: forward 5'-ACTGACCTCAAGGAGAAGTT-3'; reverse 5'GCGTTGTTCTCGGCGAG-3' [Phalaenopsis hybrid (DQ089652), Dendrobium hybrid (AY741319), and Bromheadia finlaysoniana (AF007099)]

Dfr: forward 5'-ACTCCCATGAATTTTCAATCC -3'; reverse 5'AACTCCCAAGCAGCCTTCTC-3' [B. finlaysoniana (AF007096), Dendrobium hybrid (AY741318), Cymbidium hybrid (AF017451), and Oncidium 'Gower Ramsey' (AY953939)]

RT-PCR was carried out using the Titan One Tube RT-PCR Kit (Roche Applied Science, Indianapolis). The RT-PCR control reaction was set up as described in the kit, except that the reactions were incubated at $95{ }^{\circ} \mathrm{C}$ for $10 \mathrm{~min}$ to inactivate reverse transcriptase before proceeding to the PCR step. Different cycle numbers were tested to determine the linear phase of amplification in conventional PCR. Under linear conditions, mRNA concentration could be quantified by agarose gel band intensity. The following temperatures and times resulted in linear amplification of all the genes: 30 cycles of $94{ }^{\circ} \mathrm{C}$ for $30 \mathrm{~s}, 57^{\circ} \mathrm{C}$ for $30 \mathrm{~s}, 72^{\circ} \mathrm{C}$ for s. Amplification of a Phalaenopsis Actin gene \{forward 5'-GGCACCATA CCTTCTACAACG-3'; reverse 5' -GATCGAGTTGTATGTA GTCTCATGG-3' [Phalaenopsis (U18102)]\} was used as a quantitative control between $P$. amabilis and P. schilleriana. RT-PCR was carried out multiple times with similar results.

The resulting RT-PCR fragments of expected size were cloned in the TOPO vector (Invitrogen, Carlsbad, CA) and were sequenced using the BigDye Terminator v3.1 cycle sequencing kit (Applied Biosystems, Foster City, CA). All the sequence data were edited manually based on the chromatogram output files before being compared against the GenBank nr database using blastx. DNA sequence translation and alignment were carried out using Clone Manager Suite 7 (Sci Ed Central, Cary, NC). DNA and protein sequences were compared using the standard linear and blosum 62 scoring matrix, respectively, and dendrograms were constructed using the neighbor-joining algorithm in Clone Manager.

5' - AND 3'-RACE PRIMERS AND RACE OF ANTHOCYANINReLated Мyв. Two 25-mer oligos, 5' - and 3'-RACE primers, were designed based on nine $M y b$ clones derived from $P$. schilleriana: 5RMyb: 5'-CCTTCCAGCAATTAAAGACC ACCTA-3', 3RMyb: 5' - TAGGTGGTCTTTAATTGCTGGA AGG-3'. The $5^{\prime}$ - and $3^{\prime}$-RACE-Ready cDNAs were prepared from $P$. schilleriana total RNAs using the SMART RACE cDNA Amplification Kit (Clonetech, Mountain View, CA) according to the manufacturer's protocol.

The PCR products, obtained after 30 cycles of $94^{\circ} \mathrm{C}$ for $30 \mathrm{~s}$, $57{ }^{\circ} \mathrm{C}$ for $30 \mathrm{~s}, 72{ }^{\circ} \mathrm{C}$ for s, were purified from the gel. The fragments of $5^{\prime} \operatorname{RACE}(5 R M y b)$ and $3^{\prime} \operatorname{RACE}(3 R M y b)$ were then cloned into the TOPO vector (Invitrogen) and sequenced to identify the correct clones.

Full-length PsMyb and PhredMyb cDNA isolation. Based on the sequences of six $5 R M y b$ and five $3 R M y b$ clones, a primer set flanking the start codon and terminator was designed with BamHI and $\mathrm{KpnI}$ sites added to the respective $5^{\prime}$ ends of the P. schilleriana Myb (PsMyb) primers: PsMyb5' (BamHI): 5'-GGATCCAGATGAAGCAGAGGGCTAA-3', PsMyb3' (KpnI): 5'-GGTACCTCAAAGATTTGAGTCAAA TAAGCTAC- $3^{\prime}$. 
The full-length $P s M y b s$ were amplified using this $P s M y b$ primer set and 5'-RACE-Ready cDNAs as templates. Fragments in the range of 700 to $800 \mathrm{bp}$ were obtained after 30 cycles of $94{ }^{\circ} \mathrm{C}$ for $30 \mathrm{~s}, 60^{\circ} \mathrm{C}$ for $30 \mathrm{~s}, 72{ }^{\circ} \mathrm{C}$ for $60 \mathrm{~s}$.

In addition, another $P s M y b 5^{\prime}$ primer flanking the $5^{\prime}$ untranslated region (UTR) was also devised (PsUMyb5', 5' AAGTTTATCTATCTAAGCTGGCTGA-3'). Ps UMy $b$ (fulllength $P s M y b$ plus the $5^{\prime}$-UTR) were amplified as described for the full-length $P s M y b$, except the $5^{\prime}$ primer was $P s U M y b 5^{\prime}$ instead of $P s M y b 5^{\prime}$, and the annealing temperature was lowered to $55^{\circ} \mathrm{C}$. Fragments in the range of 800 to $900 \mathrm{bp}$ were observed.

RT-PCR was carried out to amplify the full-length cDNAs from Phalaenopsis 'Everspring Fairy' (PhredMyb, harlequin mutant) using PsMyb primers. Total RNA extracted from the purple sections of the Phalaenopsis 'Everspring Fairy' was used as template for reverse transcription at $55^{\circ} \mathrm{C}$ for $30 \mathrm{~min}$, followed by 33 cycles of $94{ }^{\circ} \mathrm{C}$ for $30 \mathrm{~s}, 55^{\circ} \mathrm{C}$ for $30 \mathrm{~s}$, and $72{ }^{\circ} \mathrm{C}$ for $75 \mathrm{~s}$.

The fragments of PsMybs, PsUMybs, and PhredMybs were subsequently cloned into the TOPO vector and sequenced. Fulllength Myb cDNA fragments from P. schilleriana and Phalaenopsis 'Everspring Fairy' were identified; their GenBank accession numbers are: PsMyb1, FJ039853; PsMyb3, FJ039854; PsMyb4, FJ039855; PsMyb5, FJ039856; $P s M y b 8$, FJ039857; PsUMyb1, FJ039858; PsUMyb4, FJ039859; PsUMyb6, FJ039860; PsUMyb7, FJ039861; PsUMyb8, FJ039862; PhredMyb9, FJ039863; PhredMyb12, FJ039864; and PhredMyb13, FJ039865.

Construction of PsMybs, PsuMybs, and PhredMybs EXPRESSION CONSTRUCTS AND THEIR TRANSIENT EXPRESSION. All constructs were placed under the control of the $35 \mathrm{~S}$ promoter, with the backbone region of the plasmid vector identical to the S-R construct, as described in Ma et al. (2008). Biolistic transformation was carried out using a gene gun, and transient expression was monitored using fluorescence microscopy (Ma et al., 2008).

\section{Results and Discussion}

Comparison of genes in $\boldsymbol{P}$. amabiLIS AND $\boldsymbol{P}$. SCHILLERIANA. Anthocyanin biosynthesis requires the coordinated expression of $M y c, W d, C h s, D f r$, and $M y b$ (Griesbach, 2005). Chs and Dfr are structural genes, while $M y c, M y b$, and $W d$ are regulatory genes. Primers for Chs were largely based on the Chs gene of Phalaenopsis species (Han et al., 2006). The expected amplified fragment size is $398 \mathrm{bp}$. RT-PCR showed no detectable difference in size or expression level between the whiteflowered $P$. amabilis and purple-flowered $P$. schilleriana (Fig. 1). Sequencing three randomly selected clones of each revealed that they all shared highest homology to the Phalaenopsis chalcone synthase, although nine single-nucleotide polymorphisms (SNPs) were detected, one of which caused a nonsynonymous change in one of the clones from $P$. schilleriana (data not shown).

DFR primers were designed based on the consensus sequences derived from $D f r$ genes of four orchid genera, Bromheadia (Liew et al., 1998), Dendrobium (Mudalige-Jayawickrama et al., 2005), Cymbidium (Johnson et al., 1999), and Oncidium (Hieber et al., 2005). The primers flanked a 275-bp region in the $\mathrm{N}$-terminal domain that spans the section identified as responsible for the specificity of the substrate (Petit et al., 2007). From

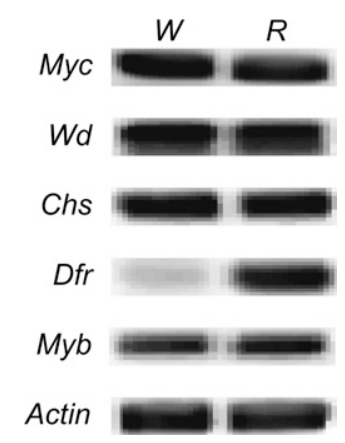

Fig. 1. RT-PCR comparison of anthocyanin regulatory/structural gene expression between Phalaenopsis amabilis (W) and Phalaenopsis schilleriana (R).

RT-PCR, there was a significant difference in $D f r$ expression between $P$. amabilis and P. schilleriana (Fig. 1). Dfr expression was significantly reduced in $P$. amabilis, resulting in an accumulation of quercetin derivatives (data not shown). Dihydroquercetin is a substrate for DFR. Therefore, the white flower color of $P$. amabilis is the result of a block in the biosynthetic pathway at DFR. We sequenced three clones from P. schilleriana and one from $P$. amabilis. The results indicated that all are very similar to the $D$ fr from Oncidium.

The R-like MYCs that function in transcriptional regulation associated with anthocyanin biosynthesis have two additional conserved regions other than the bHLH domain: a highly conserved N-terminal transactivation domain and a weakly conserved C-terminal domain (Purugganan and Wessler, 1994). Known anthocyanin-related Mycs from P. ×hybrida, A. majus, and Z. mays were used to design the RT-PCR primers. The Myc primers were based on the N-terminal region instead of the bHLH domain, which is shared by the entire MYC family because proteins sharing these additional motifs will more likely share similar functions.

RT-PCR (Fig. 1) yielded a $\approx 400$-bp fragment for $M y c$ in $P$. amabilis and $P$. schilleriana. Sequencing revealed that the fragments derived from both species were similar to a Cornus Myc-like anthocyanin regulatory protein (Fan et al., 2004). However, when compared using neighbor-joining algorithms, the randomly selected 18 clones (nine from each species) that we sequenced formed two distinct groups, with clones from each species in each group (Fig. 2). Because there was no difference in the expression of Mycs from P. amabilis and $P$. schilleriana, we concluded that Myc expression was not responsible for the lack of $D f r$ expression in $P$. amabilis.

$W d$ primers were designed within the WD motif. The expected amplified fragment of $404 \mathrm{bp}$ was found in P. amabilis and P. schilleriana RT-PCR (Fig. 1). Two clones of each were randomly selected for sequencing. Resulting sequences showed that both shared high homology to ZmPACl (Carey et al., 2004). Alignment of the four sequences displayed nine SNPs, but only one amino acid difference at the deduced protein level (data not shown). Because there was no difference in the expression of $W d s$ from $P$. amabilis and $P$. schilleriana, we concluded that $W d$ expression was not responsible for the lack of $D f r$ expression in P. amabilis.

Most plant MYB proteins are members of the R2R3 family, to which anthocyanin MYB regulatory factors belong (Stracke et al., 2007). To amplify the $M y b(s)$, we designed a set of primers based on the highly conserved region from the third helix of R2 to the third helix of R3 (residues 50-108, with 


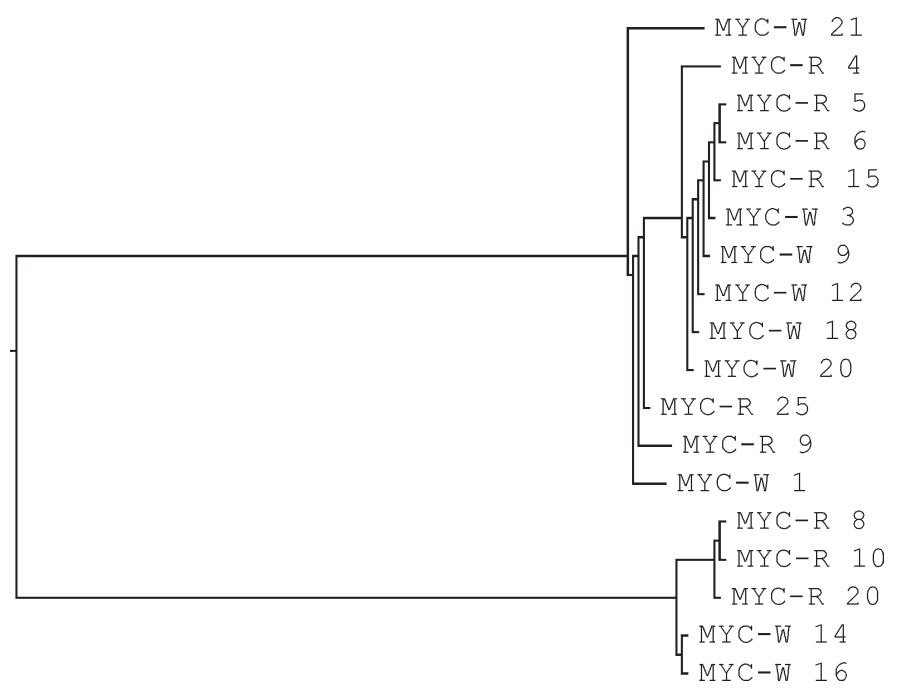

Fig. 2. Suggested pattern of relatedness of MYC deduced amino acid sequences derived from 18 randomly selected RT-PCR clones, nine each from Phalaenopsis amabilis (MYC-W 1, 3, 9, 12, 14, 16, 18, 20, and 21) and Phalaenopsis schilleriana (MYC-R 4, 5, 6, 8, 9,10,15, 20, and 25).

residue numbering based on the $Z$. mays $M y b$ gene, $C 1$ ). This region was selected because very little sequence similarity occurs outside of the highly conserved MYB domain, especially the third $\alpha$-helix within each repeat (Jiang et al., 2004).

Our RT-PCR showed no differences in expression of the expected 178-bp $M y b$ fragment between P. amabilis and P. schilleriana (Fig. 1). However, sequencing of 33 randomly selected clones with an insert size of 178 bp (18 from P. amabilis and 15 from $P$. schilleriana) revealed differences in the fragments from the two species. Overall, three clusters were exhibited when a phylogenetic tree of the 35 deduced protein sequences was constructed (Fig. 3). The Z. mays $\mathrm{Cl}$, Pl, and $\mathrm{P}$ proteins were used as landmarks. Two of the groups, which possessed highest homology to DwMyb8 or ZmP, contain clones from P. schilleriana and P. amabilis. DwMyb8 and $\mathrm{ZmP}$ are flavonol-specific regulators (Mehrtens et al., 2005). DwMYB8 was reported to be constitutively expressed in Dendrobium orchid leaves, stems, and flowers at different stages in development (Wu et al., 2003). The third subgroup shared homology to $\mathrm{ZmPl} / \mathrm{C} 1$, the MYB specific for anthocyanin biosynthesis. All nine sequences in this group were from P. schilleriana. OgMYB1 from Oncidium orchid flowers also shared homology with ZmP1/C1 (Chiou and Yeh, 2008).

The $M y b$ gene family is the one of largest regulatory gene families in plants. Only a few members of this family regulate flavonoid biosynthesis (Stracke et al., 2007). Because we used primers derived from the most conserved region, amplification of different classes of MYBs was expected. It appears that $P$. amabilis lacks anthocyanin-specific MYB expression because only the MYBs from P. schilleriana shared homology with the anthocyanin-specific $\mathrm{ZmPl} / \mathrm{Cl}$ cluster. Even though a band was amplified with the Myb primers from P. amabilis, it did not correspond to an anthocyanin-specific $M y b$. Because there was a difference in the expression of the anthocyaninspecific Myb from P. amabilis and P. schilleriana, we hypothesized that $M y b$ expression was related to the much reduced $D f r$ expression in P. amabilis, and cloned the complete $M y b$ gene for transient expression.

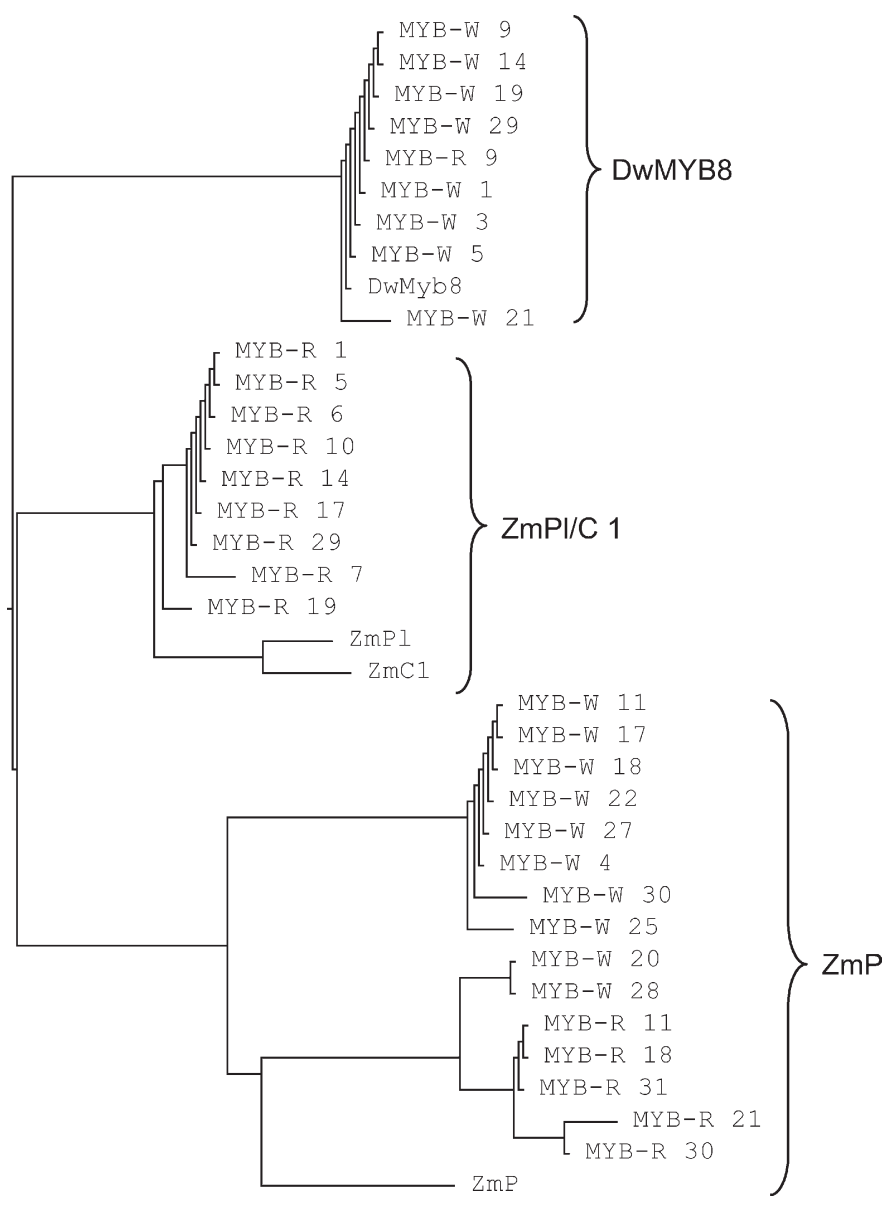

Fig. 3. Suggested pattern of relatedness of MYB deduced amino acid sequences derived from RT-PCR clones of Phalaenopsis amabilis (MYB-W 1, 3, 4, 5, 9, $11,14,17,18,19,20,21,22,25,27,28,29$, and 30), Phalaenopsis schilleriana (MYB-R 1, 5, 6, 7, 9, 10, 11, 14, 17, 18, 19, 21, 29, 30, and 31), Zea mays (ZmP, ZmPl, and $\mathrm{ZmC1}$ ), and Dendrobium species. (DwMYB8).

IsOLATION OF ANTHOCYANIN-RELATED MYB RACE CLONES AND 5' -UTR CHARACTERIZATION. To identify a region specific to $P$. schilleriana, we aligned anthocyanin-specific $M y b$ sequences from $P$. schilleriana and flavonol-related (ZmP class) $M y b$ from $P$. amabilis (Fig. 4). The region unique to P. schilleriana (25 bp, 261-285 bp from the start codon) was used to design primers for the $5^{\prime}$ - and $3^{\prime}$ - rapid amplification of cDNA ends (RACE). Using RACE-ready first-strand cDNA and appropriate $5^{\prime}$ - or $3^{\prime}$-RACE primers, we successfully amplified the corresponding RACE fragments.

Sequences from six 5 '-RACE clones $(5 R M y b)$ and five $3^{\prime}$ RACE clones $(3 R M y b)$ from $P$. schilleriana were obtained. A Blast and an open reading frame search against GenBank revealed the start and stop codon of the $M y b$ genes (Fig. 5).

Sequences of the $5^{\prime}$-UTR regions of the $5 R M y b$ clones have very distinctive characteristics: 1 ) the suboptimum start codon context (CAGATGA); the 5'-UTR region contains another upstream start codon that is not functional based upon the transcript and protein sequences of the functional $M y b$ and MYB; 2) a perfect repeat in the start codon region; and 3) various lengths of trinucleotide (CAG) repeats in the $5^{\prime}$ UTR (Fig. 6).

Among the six $5 R M y b$ clones, all contained a CAG triplet SSR with repeat numbers varying from 3 to 26 . It has been 
myb-r29 aactccttggcaataggtggtctttaattgctggaaggttgcctggtcgaacagataatgagatcaagaactactgga myb-r10 aactccttggcaataggtggtctttaattgctggaaggttgcctggtcgaacagataatgagatcaagaactactgga myb-r1 aactccttggcaataggtggtctttaattgctggaaggttacccggtcgaacggataatgagatcaagaactactgga myb-r5 aactccttggcaataggtggtctttaattgctggaaggttacccggtcgaacggataatgagatcaagaactactgga myb-r6 aactccttggcaataggtggtctttaattgctggaaggttacccggtcgaacggataatgagatcaagaactactgga myb-r17 aactccttggcaataggtggtctttaattgctggaaggttacccggtcgaacggataatgagatcaagaactactgga myb-r 7 aactccttggcaataggtggtctttaattgctggaaggttacccggtcgaacagataatgagatcaagaactactgga myb-r14 aactccttggcaataggtggtctttaattgctggaaggttacccggtcgaacagataatgagatcaagaactactgga myb-r19 gactccttggcaataggtggtctttaattgctggaaggttacccggtcgaacagacaatgagatcaagaactactgga myb-w11 ccaccattggaaacaggtggtccatcatagccggtcaactccccggccgaaccgacaacgagatcaagaactactgga myb-w17 ccaccattggaaacaggtggtccatcatagccggtcaactccccggccgaaccgacaacgagatcaagaactactgga myb-w18 ccaccattggaaacaggtggtccatcatagccggtcaactccccggccgaaccgacaacgagatcaagaactactgga myb-w22 ccaccattggaaacaggtggtccatcatagccggtcaactccccggccgaaccgacaacgagatcaagaactactgga myb-w27 ccaccattggaaacaggtggtccatcatagccggtcaactccccggccgaaccgacaacgagatcaagaactactgga myb-w4 ccaccattggaaacaggtggtccatcatagccggtcaactccccggccgaaccgacaacgagatcaagaactactgga

Fig. 4. The alignment of nine $M y b$ sequences from Phalaenopsis schilleriana (Myb-R 1, 5, 6, 7, 10, 14, 17, 19, and 29) and six $M y b$ sequences from Phalaenopsis amabilis ( $M y b-W 4,11,17,18,22$, and 27) revealed a region with high homology (underlined) among the Myb-r clones. This region was used to design 5' - and 3' -RACE primers.

5 RMyb14

5 RMyb1 6 $5 \mathrm{RMyb} 5$

5 RMyb22

5RMyb3

5RMyb1 5

3RMyb 50 3RMyb 51

3RMyb23

3RMyb1 4

3RMyb4 9 atgaagcagagggctaattgtaatgaggatggactgaacaagggagcttggactgctgagg atgaagcagagggctaattgtaatgaggatggactgaacaagggagcttggactgctgagg atgaagcagagggctaattgtaatgaggatggactgaacaagggagcttggactgccgagg atgaagcagagggctaattgtaatgaggatggactgaacaagggagcttggactgccgagg atgaagcagagggctaattgtaatgaggatggactgaacaagggaacttgggctgctgagg atgaagcagagggctaattgtaatgaggatggactgaacaagggaacttggactgctgagg

Fig. 5. Sequences from six 5'-RACE clones ( $5 R M y b)$ and five 3 '-RACE clones (3RMyb) showing the start (atg) and stop codons (tga), respectively, and their flanking sequences (underlined) used for designing primers for isolating the full-length Myb clones in Phalaenopsis schilleriana.

reported that, depending on the nature of the genes, a positive or negative correlation exists between the number of repeats and the transcription activities (Shinagawa et al., 1997). That is, both deletion and expansion of the repeat can either increase or impede gene expression.

Two CAG units were also present, one immediately upstream and one three nucleotides downstream of the start codon. SSRs in the $5^{\prime}$-UTRs have been reported to serve as protein-binding sites, hence regulating gene translation (Paul et al., 1987). We speculate that the CAG repeat binding site in our $M y b 5^{\prime}$-UTR might not only influence its translation activity, but also dictate its proper start codon.

The location of the start codon of the $5 R M y b$ clones was predicted based on GenBank searches using blastx. A stretch of nine nucleotides from the -9 position to the -1 position in the $M y b s$, ATGAAGCAG, is identical to that from the +1 position to the +9 position, which means there is an untranslated start codon (uAUG) six nucleotides upstream of the real start codon. The presence of uAUG has been shown to decrease the mRNA translational efficiency (Kochetov et al., 2002).

Joshi et al. (1997) extensively surveyed over 5000 unbiased plant gene transcripts and discovered that more than $80 \%$ of them have a purine (A or $\mathrm{G}$ ) at the -3 position and $\mathrm{G}$ at the +4 position. It was concluded that deviation from this norm might result in lower protein translation efficiency. In our case, both criteria were violated, with a pyrimidine at the -3 and a non-G base at the +4 positions.

Joshi's survey also found that those transcripts with suboptimal start codon contexts often encode transcription factors, regulatory proteins/signal transducers, etc. Therefore, such digression of our $M y b$ genes from the consensus sequence might provide an alternative regulatory mechanism to control the cellular availability of anthocyanin-related $M y b s$ to ensure the precise control of its cellular concentration.

ISOLATION OF FULL-LENGTH ANTHOCYANIN-RELATED Mybs AND THEIR TRANSIENT EXPRESSION. Alignment of sequences in $5^{\prime}-$ and $3^{\prime}$ RACE groups revealed consensus sequences that allowed us to design primers to amplify the full-length $M y b$ cDNAs with the 5'-UTR (PsU$M y b)$ and without the 5'-UTR $(P s M y b)$. We isolated five fulllength $P s M y b$ s and seven full-length $M y b s$ with $5^{\prime}$-UTR $(P s U M y b)$. All encoded predicted proteins of 239 amino acids.

The $5^{\prime}$-UTR regions of the $P_{S} U$ $M y b$ clones revealed the same pattern as in the $5 R M y b$ clones (Fig. 6). Interestingly, we found two different types of $M y b$ DNA sequences that were associated with the shorter (PsUMyb-3, -5, and -7) or longer repeats ( $P S U M y b-1,-4,-6$, and -8), respectively. Among the three $P_{S} U$ $M y b$ s with shorter repeats, two had a premature stop codon due to a point mutation $(\mathrm{CGA} \rightarrow \mathrm{TGA}, P s U M y b-5)$ or frame shift that resulted from an insertion ( $P S U M y b-3$, data not shown).

Alignment of both DNA and predicted protein sequence (not including the two with premature stop codons) revealed two different types of $M y b s$ (Fig. 7). One group consisted of $P s M y b-3$, -8 and PSUMyb-1, 4, 6, and -8; the other, PsMyb-1, -4, -5, and $P S U M y b-7$. It seemed there was a correlation between difference in the length of trinucleotide (CAG) repeats in $5^{\prime}-\mathrm{UTR}$ and difference in the gene coding sequences, with the longer length of the repeat $(>20)$ associated with the former group and the shorter length of the repeats with the latter group. The most divergent area was located near the carboxyl-terminal, between amino acids 121 and 185 (Fig. 7). This stretch of 65 amino acids only shared $68 \%$ to $74 \%$ homology, compared with $95 \%$ to $98 \%$ in the most conserved MYB region between amino acids 50 and 108 (Fig. 7).

We previously developed an in vivo functional assay system to monitor $M y c / M y b$ gene expression using particle bombardment to introduce $M y c / M y b$ constructs into white $P$. amabilis petal tissue (Griesbach and Klein, 1993; Ma et al., 2008). We used this system to study whether the two different types of $M y b s$ described above were able to induce anthocyanin synthesis. Transient expression of $P S U M y b-6$ and -7, and $P s M y b-3,-4$, -5 , and -8 in Phalaenopsis indicated they were all able to induce anthocyanin production in the white petals. The bombardment by the $P s U M y b-6+L c(Z$. mays $M y c)$ construct is shown in Fig. 8. The other constructs had similar expression patterns.

The structural gene $D f r$ was expressed differentially in the petals and sepals of $P$. amabilis and $P$. schilleriana. Dfr was significantly reduced in the white petals and sepals of $P$. amabilis. In $P$. amabilis and $P$. schilleriana, anthocyanin-specific $M y c$ 


\begin{tabular}{|c|c|c|c|c|}
\hline & $A G[C A G]_{3}$ & AGCAAAGATGAAGCAG & ATGAAGCAGAGGGCTAAT & (5RMyb22) \\
\hline AAGTTTATCTATCTAAGCTGGCTGAGGT & {$[C A G]_{4}$} & AAGCAAAGATGAAGCAG & ATGAAGCAGAGGGCTAAT & \\
\hline AAGTTTATCTATCTAAGCTGGCTGAGGTG & $\mathrm{AG}[C A G]_{26}$ & AGGCAAAGATGAAGCAG & ATGAAGCAGAGGGCTAAT & (5 \\
\hline AGTTTATCTATCTAAGCTGGCTGAGGT & {$[C A C$} & AAGCAAAGATGAAGCAG & ATGAAGCAGAGGGCTAAT & $(\mathrm{P}$ \\
\hline AAGTTTATCTATCTAAGCTGGCTGAGGT & {$[C$} & AAGCAAAGATGAAGCAG & ATGAAGCAGAGGGCTAAT & \\
\hline AAGTTTATCTATCTAAGCTGGCTGAGGT & {$[C A C$} & AAGCGAAGATGAAGCAG & ATGAAGCAGAGGGCTAAT & \\
\hline AAGTTTATCTATCTAAGCTGGCTGAGGTC & $\mathrm{AAG}[C A G]_{24}$ & AGGCAAAGATGAAGCAG & ATGAAGCAGAGGGCTAAT & i \\
\hline AAGTTTATCTATCTAAGCTGGCTGAGGTC & $\mathrm{B} A \mathrm{G}[\mathrm{CAG}]_{24}$ & AGGCAAAGATGAAGCAG & ATGAAGCAGAGGGCTAAT & \\
\hline AAGTTTATCTATCTAAGCTGGCTGAGGT & L 200 & 20 & ATGAAGCAG & \\
\hline
\end{tabular}

Fig. 6. Sequence of the $5^{\prime}$-UTR region of $5 R M y b$ and $P S U M y b$ clones from Phalaenopsis schilleriana. Distinct characteristics include a suboptimum start codon (CAGATGA), a perfect repeat (AGATGAAG) in the start codon region, and various lengths of trinucleotide (CAG) repeats.

\begin{abstract}
PSMYB8
PSUMYB4

PSUMYB6

PSUMYB1

PSUMYB8

PhredMYB9

PsMYB3

PSMYB1

PSUMYB7

PSMYB4

PSMYB5

PhredMYB13

PhredMYB12

PSMYB8

PSUMYB 4

PsUMYB6

PsUMYB1

PsUMYB8

PhredMYB9

PsMYB3

PSMYB1

PSUMYB7

PSMYB4

PSMYB5

PhredMYB13

PhredMYB12

PSMYB8

PSUMYB4

PSUMYB 6

PSUMYB1

PSUMYB8

PhredMYB9

PSMYB3

PSMYB1

PSUMYB7

PSMYB4

PSMYB5

PhredMYB13

PhredMYB12

201 dsfsfddemwidglefggeinvvddydnylvcslfdsnl 201 dsfsfddemwidglefggeinvvddydnylvcslfdsnl 201 dsfsfddemwidglefggeinvvddydnylvcslfdsnl 201 dsfsfddemwidglefggeinvvddydnylvcslfdsnl 201 dsfsfddemwidglefggeinvvddydnylvcslfdsnl 201 dsfsfddemwidglefggeinvvddydnylvgslfdsnl 201 dsfsfddemwidglefggeinvvddydnylvcslfdsn 1 201 dlfsfddemwidgfefggeinvvddydndlvcslfdsnl 201 dlfsfddemwidgfefggeinvvddydndlvcslfdsn 1 201 dlfsfdemwidgfefggeinvvddydndlvcslfdsnl 201 dsfsfddemwidglefggeinvvddydndlvcslfdsn 1 200 dsfsfdemwiaglefggginvfddydndlicslfdsnl 200 dsfsfdemwiaglefggginvfddydndlicslfdsnl
\end{abstract}

1 mkqrancnedglnkgtwtaeedellasyvnavgegnwtsvpkkaglnrrgkscrlrwlnylrpnikrgnisveeeeliirlhrllgnrwsliagrlpgrt 1 mkqrancnedglnkgtwtaeedellasyvnavgegnwtsvpkkaglnrrgkscrlrwlnylrpnikrgnisveeeeliirlhrllgnrwsliagrlpgrt 1 mkgrancnedglnkgtwtaeedellasyvnavgegnwtsvpkkaglnrrgkscrlrwlnylrpnikrgnisveeeeliirlhrllgnrwsliagrlpgrt 1 mkqrancnedglnkgtwtaekdellasyvnavgegnwtsvpkkaglnrrgkscrlrwlnylrpnikrgnisveeeeliirlhrllgnrwsliagrlpgrt 1 mkqrancnedglnkgtwtaeedellasyvnavgegnwtsvpmkaglnrrgkscrlrwlnylrpnikrgnisveeeeliirlhrllgnrwsliagrlpgrt 1 mkgrancnedglnkgtwtaeedellasyvnavgegnwtsvpkkaglnrrgkscrlrwlnylrpnikrgnisveeeeliirlhrllgnrwsliagrlpgrt 1 mkqrancnedglnkgtwtaeedellasyvnavgegnwtsvpkkaglnrrgkscrlrwlnylrpnikrgnisaeeeeliirlptllgnrwsliagrlpgrt 1 mkqrancnedglnkgawtaeedellasyvnavgegnwtsvpkkaglnrrgkscrlrwlnylrpnikrgnisveeeeliirlhkllgnrwsliagrlpgrt 1 mkqrancnedglnkgawtaeedellasyvnavgegnwtsvpkkaglnrrgkscrlrwlnylrpnikrgnisveeeeliirlhkllgnrwsliagrlpgrt 1 mkqrancnedglnkgawtaeedellasyvnavgegnwtsvpkkaglnrrgkscrlrwlnylrpnikrgnisveeeeliirlhkllgnrwsliagrlpgrt 1 mkqrancnedglnkgawtaeedellasyvnavgegnwtsvpkkaglnrrgkscrlrwlnylrpnikrgnisveeeeliirlhkllgnrwsliagrlpgrt 1 mkqrakydedgvnkgawtaeedellasyvnavgegnwtsvpkkaglnrrgkscrlrwlnylrpnikrgnisveeeeliirlhkllgnrwsliagrlpgrt

1 mkqrakydedgvnkgawtaeedellasyvnavgegnwtsvpkkaglnrrgkscrlrwlnylrpnikrgnisveeeeliirlhkllgnrwsliagrlpgrt

101 dneiknywnttlskkiqtkkitinmpdikelkpksnpletnitssstpyliqtkalkctkasypshiqpstngvtqtqqisqqnfqdelmeekkdkvldc 101 dneiknywnttlskkiqtkkitinmpdikelkpksnpletnitssstpyliqtkalkctkasypshiqpstngvtqtqqisqqnfqdelmeekkdkvldc 101 dneiknywnttlskkiqtkkitinmpdikelkpksnpletnitssstpyliqtkalkctkasypshiqpstngvtqtqqisqqnfqdelmeekkdkvldo 101 dneiknywnttlskkiqtkkitinmpdikelkpksnpletnitssstpyliqtkalkctkasypshiqpstngvtqtqqisqqnfqdelmeekkdkvldc 101 dneiknywnttlskkiqtkkitinmpdikelkpksnpletnitssstpyliqtkalkctkasypshiqpstngvtqtqqisqqnfqdelmeekkdkvldo 101 dneiknywnttlskkiqtkkitinmpdikelkpksnpletnitssstpyliqtkalkctkasypshiqpstngvtqtqqisqqnfqdelmeekkdkvldc 101 dneiknywnttlskkiqtkkitinmpdikelkpksnpletnitssstpyliqtkalkctkasypshiqpstngvtqtqqisqqnfqdelmeekkdkvldc 101 dnetknywnttlskkiqtkkstinmpnikawnpktnpleanitsssisyliqtkaqnesktsnplihtlpstngvmqtoqiseqnsqdelmeekkdkvldc 101 dneiknywnttlskkiqtkkstinmpnikawnpktnpleanitsspisyliqtkaqncsktsnplhtlpstngvmqtqqiseqnsqdelmeekkdkvldc 101 dneiknywnttlskkiqtkkstinmpnikawnpktnpleanitsssisyliqtkaqncsktsnplhtlpstngvmqtqqisgqnsqdelmeekkdkvldc 101 dneiknywnttlskkiqtkkstinmpnikawnpktnpleanitsssisyliqtkaqncsktsdplhilpstngvmqtaqisqgnfqdelmeekkdkvldo 101 dneiknywnttlskkiqtkkftinmpnmkawkpksnpletkits-svsysiqtelectktsyplhvlpstsgviqtqqinkqnfqeelmeekkdkvldy 101 dneiknywnttlskkiqtkkftinmpnmkawkpknpletkits-svsysiqt telectktsyplhvlpstsgviqtqqinkqnfqeelmeekkdkvldy

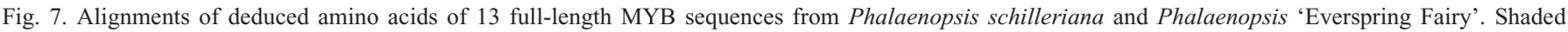
characters differ from the majority consensus sequences and dashes indicate gaps. The R2R3 motif is in bold and underlined.

and $W d$ were expressed; however, $M y b$ specific for anthocyanin biosynthesis was undetectable in $P$. amabilis petals and sepals. This suggests that the absence of $M y b$ expression is responsible for the lack of DFR and results in the absence of anthocyanin pigmentation in $P$. amabilis petals and sepals.

Even though $P$. amabilis petals and sepals do not produce functional $M y b$ and $D f r$ transcripts, functional copies of both genes must be present because $P$. amabilis flowers are not totally white; the labellum contains areas of deep anthocyanin pigmentation. Because the labellum produces anthocyanins, the lack of anthocyanin in the petals and sepals must be the result of differential tissue-specific gene expression.

Comparisons of anthocyanin-related $M y b$ gene expression between $P$. schilleriana (solid purple) and P. amabilis (albescent phenotype) are between genetically different species. Neither an albescent $P$. schilleriana nor a solid purple $P$. amabilis exist. Even though flowers of $P$. amabilis (albescent phenotype) contain pigmented areas in the labellum, it is not possible to isolate quality RNA from labellum tissue. Within flowers of the Phalaenopsis 'Everspring Fairy' (harlequin 


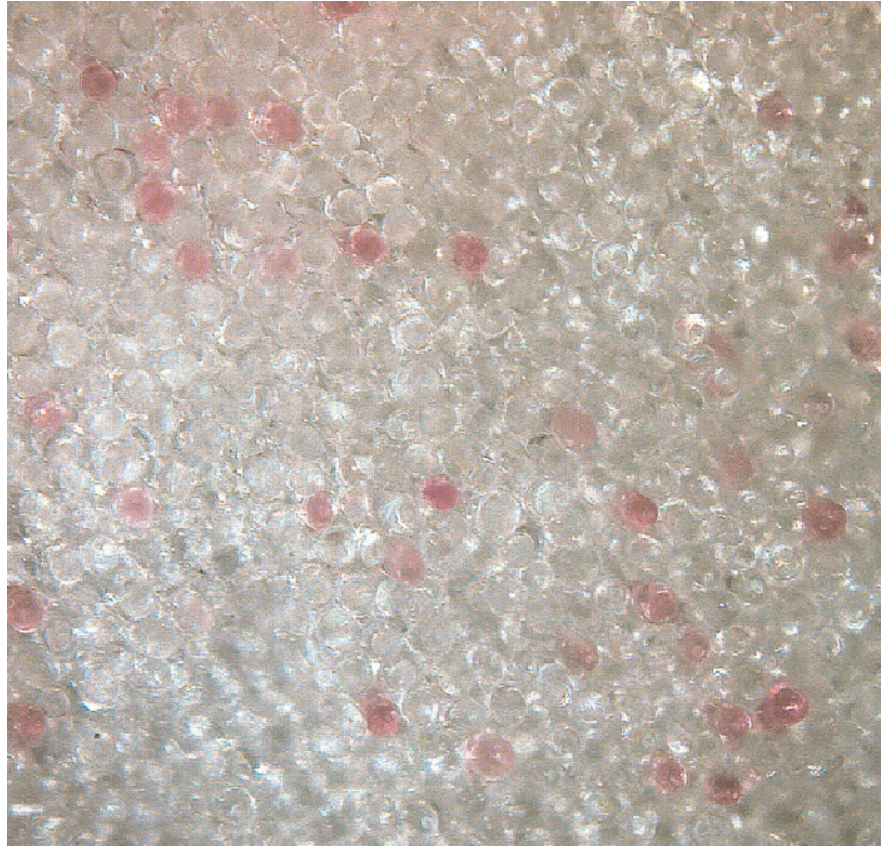

Fig. 8. Transient expression of PsUMyb-6 coupled with the Zea mays Myc Lc gene as described previously (Ma et al., 2008) yields anthocyanin expression in bombarded cells.

phenotype), quality RNA can be isolated from both the pigmented and unpigmented areas within the petal. Therefore, harlequin flowers are ideal to evaluate anthocyanin-related $M y b$ gene expression within genetically identical but differently pigmented tissue.

To further confirm that the primers we used to isolate the full-length Mybs from P. schilleriana are indeed anthocyaninrelated, we carried out RT-PCR using PsMyb primers on RNAs extracted from purple and white sections of the harlequin mutant Phalaenopsis 'Everspring Fairy'. With PsMyb primers, a fragment of 700 to $800 \mathrm{bp}$ was only present in the purple section (Fig. 9). This fragment was isolated and several randomly selected clones were sequenced. A sequence homology search revealed that three clones (PhredMyb-9, -12, and -13) were full-length Mybs.

PhredMybs were then compared with PsMYBs and PsUMybs (Fig. 7). One noticeable difference was a one-amino acid deletion in PhredMyb-12 and -13 with respect to amino acid 146 of PhredMyb-9 and PsMYBs/PsUMybs. The length of the MYB domain is expected to be highly conserved because it could affect Myb DNA-binding activity. However, the effects of the altered length outside of the MYB domain are not clear. PhredMyb 9 and PhredMyb 13 were made into expression constructs for transient expression. These assays showed that both were able to induce anthocyanin production (data not shown). Therefore, we concluded that the PsMyb primers were able to amplify anthocyanin-related full lengths Mybs and Phalaenopsis 'Everspring Fairy' petal color pattern was likely due to the differential expression of anthocyanin Mybs.

$M y b$ expression is also responsible for anthocyanin pigmentation in P. Xhybrida and Oncidium flowers. In P. ×hybrida, a mutation in $A n 2(M y b)$ reduces the expression of $A n 6(D f r)$ and results in white flowers (Quattrocchio et al., 1999). In Oncidium 'Gower Ramsey', the absence of anthocyanin pigmentation

Fig. 9. Agarose gel electrophoresis separation of the full-length Myb (700-800 bp) RT-PCR products derived from total RNA extracted from white and purple sections from petals of Phalaenopsis 'Everspring Fairy'. Lanes 3 and $4=$ RT-PCR results from white and purple sections, respectively; lanes 1 and $2=$ RT-PCR control of the corresponding samples without the reverse transcription step; lane 5 = 100-bp DNA ladder (Invitrogen, Carlsbad, CA).

was attributed to lack of DFR and CHI (chalcone isomerase) expression (Chiou and Yeh, 2008). Several Mybs were expressed in Oncidium flowers, one of which, $O g M y b B 1$, was associated with anthocyanin production.

Comparison of Phalaenopsis Mybs. Phalaenopsis MYBs' predicted protein sequences (Fig. 7) are consistent with the characterization of R2R3-Mybs, which are highly conserved in the MYB domain and strikingly divergent outside the MYB domain. The hallmark of plant R2R3-MYB domains is the number of Trp residues (three in R2 and two in R3) and their corresponding positions in each repeat. The three Trps in R2 of all the Phalaenopsis MYBs are spaced evenly 20 amino acids apart at positions 17,37 , and 57, while the two Trps are positioned 19 amino acids apart in R3. The Trp residues are part of a hydrophobic core to facilitate DNA binding. In contrast, the animal R2R3-MYB domain has three Trps in R3. In all 10 of the Phalaenopsis MYBs, the first Trp (at position 70) is replaced with Ile. This is different from Dendrobium and A. thaliana MYBs. Among the 21 partial Dendrobium MYBs analyzed (Wu et al., 2003), only two of them substitute Trp-70 with Ile, and the predominant substitution amino acid is Phe. A similar pattern is seen in A. thaliana Mybs (Stracke et al., 2007). Nevertheless, all use a hydrophobic amino acid to replace the Trp.

The R2 and R3 repeat is joined by a linker composed of about nine amino acids (63-71). The linker is believed to provide flexibility to position the DNA-recognition helices on their target DNA sequence, but the level of sequence conservation requirement for this region is unknown (Rabinowicz et al., 1999). At position 63, a Pro residue was found in both animal and plant Myb domains. It was highly conserved with few exceptions. This Pro can be replaced by Ala, Arg, and Ser, which resulted in so-called A-, R-, and S-type Mybs, respectively (Jiang et al., 2004). The Phalaenopsis MYBs isolated thus far are P-type Mybs, which have Pro63.

Key Role of ANTHOCYANIN Mybs in CONDITIONING PIGMENTATION. It is widely accepted that the anthocyanin biosynthesis pathway is regulated largely by the MYB-MYCWD regulatory protein complex (Koes et al., 2005). WDs serve as a platform for protein-protein interactions with MYCs, which, in turn, recruit MYBs to form a transcription complex. This complex includes various members of the MYB, MYC, and WD families. The working hypothesis is that the ubiquitous WD interacts with multiple MYCs, and each MYC enlists different MYBs that eventually bind to the unique target gene to initiate gene expression. 
To gain some insight as how this triad regulates anthocyanin expression in Phalaenopsis, we carried out similar RT-PCR surveys on the anthocyanin gene $(M y c, W d, C h s$, and $D f r$ ) expression between white and purple sections of the Phalaenopsis 'Everspring Fairy' petals as well. We used the same primers designed for the expression survey with $P$. amabilis and $P$. schilleriana. The RT-PCRs indicated that in addition to anthocyanin-specific $M y b, D f r$ transcripts were present in the purple, but not in the white, sectors (data not shown). There was no differential expression of $C h s, W d$, and $M y c$ between the purple and white sectors (data not shown). These results are in agreement with our results from $P$. amabilis and $P$. schilleriana.

Based on the sequence data from three different Phalaenopsis species, WDs had the least variation at the protein level. This observation could be partially explained by the network regulation model because MYB and MYC together appeared to be sufficient to dictate the specificity of the regulatory complex. The RT-PCR result, however, strongly indicated that it is MYB that plays the key role in determining the anthocyanin production, with $D$ fr its potential targeted structural gene.

Plant MYBs have proven to be encoded by a large gene family in $Z$. mays ( $>80 \mathrm{MYBs}$ ) and $A$. thaliana (125 MYBs). It appears that this could be true in Phalaenopsis as well based on partial Myb sequences and several full-length cDNAs of anthocyanin-specific Mybs isolated from P. schilleriana. The $5^{\prime}$-UTR regions of these sequences displayed distinctive molecular features that might provide clues as to how the anthocyanin-specific regulatory gene $M y b$ is itself regulated. Another possible mechanism for the regulation of $M y b$ expression includes micro-RNA. The occurrence of virus-induced color break in some orchids is suggestive of interference in micro-RNA regulation due to interactions with viral suppressors of RNA silencing (Teycheney and Tepfer, 2001). Further studies are underway to identify anthocyanin-specific micro-RNAs.

\section{Literature Cited}

Baudry, A., M. Caboche, and L. Lepiniec. 2006. TT8 controls its own expression in a feedback regulation involving TTG1 and a homologous MYB and bHLH factors, allowing a strong and cell-specific accumulation of flavonoids in Arabidopsis thaliana. Plant J. 46:768-779.

Brevitz, J.O., Y. Xia, J. Blount, R.A. Dixon, and C. Lamb. 2000. Activation tagging identifies a conserved MYB regulator of phenylpropanoid biosynthesis. Plant Cell 12:2383-2394.

Carey, C.C., J.T. Strahle, D.A. Selinger, and V.L. Chandler. 2004. Mutations in the pale aleurone colorl regulatory gene of the Zea mays anthocyanin pathway have distinct phenotypes relative to the functionally similar TRANSPARENT TESTA GLABRA1 gene in Arabidopsis thaliana. Plant Cell 16:450-464.

Chandler, V.L., J.P. Radicella, T.P. Robbins, J. Chen, and D. Turks. 1989. Two regulatory genes of the maize anthocyanin pathway are homologous: Isolation of B-utilizing $\mathrm{R}$ genomic sequences. Plant Cell 1:1175-1183.

Chiou, C.-Y. and K.-W. Yeh. 2008. Differential expression of MYB gene (OgMYB1) determines color patterning in floral tissue of Oncidium Gower Ramsey. Plant Mol. Biol. 66:379-388.

Cone, K.C., F.A. Burr, and B. Burr. 1986. Molecular analysis of the maize anthocyanin regulatory locus $C 1$. Proc. Natl. Acad. Sci. USA 83:9631-9635.

deVetten, N., F. Quattrocchio, J. Mol, and R. Koes. 1997. The an11 locus controlling flower pigmentation in Petunia encodes a novel WD-repeat protein conserved in yeast, plants and animals. Genes Dev. 11:1422-1434.

Fan, C., M.D. Purugganan, D.T. Thomas, B.M. Wiegmann, and Q.-Y. Xiang. 2004. Heterogeneous evolution of the Myc-like anthocyanin regulatory gene and its phylogenetic utility in Cornus L. Mol. Phylogenet. Evol. 33:580-594.

Griesbach, R.J. 2005. Biochemistry and genetics of flower color. Plant Breed. Rev. 25:89-114.

Griesbach, R. and T. Klein. 1993. In situ genetic complementation of a flower color mutant in Doritis pulcherrima (Orchidaceae). Lindleyana 8:223-226.

Grotewold, E., M.B. Sainz, L. Tagliani, J.M. Hernandez, B. Bowen, and V.L. Chandler. 2000. Identification of the residues in the $M y b$ domain of maize $\mathrm{C} 1$ that specify the interaction with the bHLH cofactor R. Proc. Natl. Acad. Sci. USA 97:13579-13584.

Han, Y.Y., F. Ming, W. Wang, J.W. Wang, M.M. Ye, and D.L. Shen. 2006. Molecular evolution and functional specialization of chalcone synthase superfamily from Phalaenopsis orchid. Genetica 128:429438.

Hartmann, U., M. Sagasser, F. Mehrtens, R. Stracke, and B. Weisshaar. 2005. Differential combinatorial interactions of cis-acting elements recognized by R2-R3-Myb, BZIP and BHLH factors control light responsive and tissue-specific activation of phenylpropanoid biosynthesis genes. Plant Mol. Biol. 57:155-171.

Hernandez, J.M., G.F. Heine, N.G. Irani, A. Feller, M.-G. Kim, T. Matulnik, V.L. Chandler, and E. Grotewold. 2004. Different mechanisms participate in the R-dependent activity of the R2R3 MYB transcription factor C1. J. Biol. Chem. 279:48205-48213.

Hieber, D.A., R.G. Mudalige-Jayawickrama, and A.R. Kuehnle. 2005. Color genes in the orchid Oncidium Gower Ramsey: Identification, expression, and potential genetic instability in an interspecific cross. Planta 223:521-531.

Irani, N., J.M. Hernandez, and E. Grotewold. 2003. Regulation of anthocyanin pigmentation. Recent Adv. Phytochem. 38:59-78.

Jiang, C., X. Gu, and T. Peterson. 2004. Identification of conserved gene structures and carboxy-terminal motifs in the $M y b$ gene family of Arabidopsis and Oryza sativa indica. Genome Biol. 5:R46.

Johnson, E.T., Y. Hankuil, B. Shin, B.-J. Oh, H. Cheong, and G. Choi. 1999. Cymbidium hybrida dihydrofavonol 4-reductase does not efficiently reduce dihydrokaempferol to produce orange pelargonidin-type anthocyanins. Plant J. 19:81-85.

Joshi, C.P., H. Zhou, X. Huang, and V.L. Chiang. 1997. Context sequences of translation initiation codon in plants. Plant Mol. Biol. 35:993-1001.

Kochetov, A.V., O.A. Sirnik, I.B. Rogosin, G.V. Glazko, M.L. Komarova, and V.K. Shumny. 2002. Contextual features of higher plant mRNA 5' -untranslated regions. Mol. Biol. 36:510-516.

Koes, R., W. Verweij, and F. Quattrocchio. 2005. Flavonoids: A colorful model for the regulation and evolution of biochemical pathways. Trends Plant Sci. 10:236-242.

Liew, C.F., C.S. Loh, C.J. Goh, and S.H. Lim. 1998. The isolation, molecular characterization and expression of dihydroflavonol 4reductase cDNA in the orchid, Bromheadia finlaysoniana. Plant Sci. 135:161-169.

Ludwig, S.R., L.F. Habera, S.L. Dellaporta, and S.R. Wessler. 1989. $\mathrm{Lc}$, a member of the maize $\mathrm{R}$ gene family responsible for tissuespecific anthocyanin production, encodes a protein similar to transcriptional activators and contains the myc-homology region. Proc. Natl. Acad. Sci. USA 86:7092-7096.

Ma, H., M. Pooler, and R. Griesbach. 2008. Ratio of $M y c$ and $M y b$ transcription factors regulates anthocyanin production in orchid flowers. J. Amer. Soc. Hort. Sci. 133:133-138.

Mehrtens, F., H. Kranz, P. Bednarek, and B. Weisshaar. 2005. The Arabidopsis transcription factor MYB12 is a flavonol-specific regulator of phenylpropanoid biosynthesis. Plant Physiol. 138:1083-1096.

Mudalige-Jayawickrama, R.G., M.M. Champagne, A.D. Hieber, and A.R. Kuehnle. 2005. Cloning and characterization of two anthocyanin biosynthetic genes from Dendrobium orchid. J. Amer. Soc. Hort. Sci. 130:611-618.

Paul, A.L., V. Vasil, I.K. Vasil, and R.J. Ferl. 1987. Constitutive and anaerobically induced DNase-I-hypersensitive sites in the 5' 
region of the maize Adh1 gene. Proc. Natl. Acad. Sci. USA 84:799803.

Paz-Ares, J., U. Ghosal, U. Wienand, P. Peterson, and H. Saedler. 1987. The regulatory $\mathrm{c} 1$ locus of Zea mays encodes a protein with homology to myb proto-oncogene products and with structural similarities to transcriptional activators. EMBO J. 6:3553-3558.

Petit, P., T. Granier, B.L. d'Estaintot, C. Manigand, K. Bathany, J.M. Schmitter, V. Lauvergeat, S. Hamdi, and B. Gallois. 2007. Crystal structure of grape dihydroflavonol 4-reductase, a key enzyme in flavonoid biosynthesis. J. Mol. Biol. 368:1345-1347.

Purugganan, M.D. and S.R. Wessler. 1994. Molecular evolution of the plant R regulatory gene family. Genetics 138:849-854.

Quattrocchio, F., J. Wing, K. Woude, E. Souer, N. de Vetten, J. Mol, and R. Koes. 1999. Molecular analysis of the anthocyanin 2 gene of petunia and its role in the evolution of flower color. Plant Cell 11:1433-1444.

Rabinowicz, P.D., E.L. Braun, A.D. Wolfe, B. Bowen, and E. Grotewold. 1999. Maize R2R3 Myb genes: Sequence analysis reveals amplification in higher plants. Genetics 153:427-444.

Radicella, J.P., D. Turks, and V.L. Chandler. 1991. Cloning and nucleotide sequence of a cDNA encoding B-Peru, a regulatory protein of the anthocyanin pathway from maize. Plant Mol. Biol. 17:127-130.

Ramsay, N.A. and B.J. Glover. 2005. MYB-bHLH-WD40 protein complex and the evolution of cellular diversity. Trends Plant Sci. 10:63-70.

Sainz, M.B., E. Grotewold, and V.L. Chandler. 1997. Evidence for direct activation of an anthocyanin promoter by the maize $\mathrm{C} 1$ protein and comparison of DNA binding by related Myb domain proteins. Plant Cell 9:611-625.

Shinagawa, T., N. Ishiguro, M. Horiuchi, T. Matsui, K. Okada, and M. Shinagawa. 1997. Deletion of c-myb exon 9 induced by insertion of repeats. Oncogene 14:2775-2783.

Smith, T.F. 1999. The WD repeat: A common architecture for diverse functions. Trends Biochem. Sci. 24:181-185.

Stracke, R., H. Ishihara, G. Huep, A. Barsch, F. Mehrtens, K. Niehaus, and B. Weisshar. 2007. Differential regulation of closely related R2R3-MYB transcription factors controls flavonol accumulation in different parts of the Arabidopsis thaliana seedling. Plant J. 50:660677.

Tang, C.Y. and W.H. Chen. 2007. Breeding and development of new varieties in Phalaenopsis, p. 1-22. In: W.H. Chen and H.H. Chen (eds.). Orchid biotechnology. World Scientific, Singapore.

Teycheney, P.Y. and M. Tepfer. 2001. Virus-specific spatial differences in the interference with silencing of the chs-A gene in nontransgenic petunia. J. Gen. Virol. 82:1239-1243.

Wu, X.M., S.H. Lim, and W.C. Yang. 2003. Characterization, expression and phylogenetic study of R2R3-MYB genes in orchid. Plant Mol. Biol. 51:959-972.

Zhang, P., S. Chopra, and T. Peterson. 2000. A segmental gene duplication generated differentially expressed myb-homologous genes in maize. Plant Cell 12:2311-2322.

Zimmermann, I.M., M.A. Heim, B. Weisshaar, and J.F. Uhrig. 2004. Comprehensive identification of Arabidopsis thaliana MYB transcription factors interacting with R/B-like BHLH proteins. Plant J. 40:22-34. 